\title{
Aprendizaje autónomo del alumnado de Ciencia Política en sus portafolios
}

\author{
José Francisco JiMÉnEZ DÍAZ \\ Universidad Pablo de Olavide \\ josefco@upo.es
}

Recibido: $27 / 07 / 2012$

Aceptado: 08/10/2012

\begin{abstract}
Resumen
Este artículo muestra la experiencia de aprendizaje autónomo de estudiantes universitarios en una asignatura de Ciencia Política perteneciente al Espacio Europeo de Educación Superior (EEES). El portafolio basado en lecturas y escritos reflexivos es la herramienta docente aplicada en dicha experiencia. Por ello, se analizan los discursos de los portafolios de diez alumnos en los que se evidencian sus diversos aprendizajes autónomos. Tales aprendizajes se desarrollan en un proceso de enseñanza-aprendizaje enfocado hacia la elaboración de escritos reflexivos por los estudiantes. Así, estos últimos son formados en las prácticas discursivas de la Ciencia Política a través de lecturas de reflexión claves que, a su vez, permiten a los estudiantes construir sus conocimientos.
\end{abstract}

Palabras clave: Aprendizaje autónomo, portafolio, estudiante, escritura.

\section{Autonomous learning of student of Political Science in their portfolios}

\begin{abstract}
This article shows the autonomous learning experience of university students in a subject of Political Science belonging to European Higher Education Area. The teaching tool applied in this experience is the portfolio based on reflective readings and writings. Consequently, I analyze the discourses of ten student portfolios, in which the autonomous learning is showed. In this case, the autonomous learning involves a teaching-learning process focused on the development of reflective writings by students. So, the students are formed in the discursive practices of Political Science through key reflective readings that, in turn, allow them to build their knowledge.
\end{abstract}

Key words: Autonomous learning, portfolio, student, writing.

Referencia normalizada

JIMÉNEZ DÍAZ, José Francisco (2012): “Aprendizaje autónomo del alumnado de Ciencia Política en sus portafolios”. Estudios sobre el mensaje periodístico. Vol. 18, núm. especial noviembre, págs.: 543550. Madrid, Servicio de Publicaciones de la Universidad Complutense.

Sumario: 1. Introducción. 2. Metodología. 3. Desarrollo. 4. Conclusiones. 5. Referencias bibliográficas.

\section{Introducción}

El Espacio Europeo de Educación Superior (EEES) centra su atención en el aprendizaje de los alumnos, lo cual supone que los docentes han de abordar cambios profundos en las maneras de hacer y de entender su actividad, al tiempo que los primeros deben asumir un papel más activo y participativo en la construcción de sus conocimientos. Así, una de las claves del EEES es que los docentes enseñen "a aprender a aprender" (Colen et al., 2006: 18) a los estudiantes para así poder brindarles una formación integral (Benito y Cruz, 2005: 15). Ello supone que la función del docente no ha de limitarse a transmitir conocimientos, sino "ayudar al alumno a aprender con el objetivo de que él luego sea capaz de hacerlo autónomamente" (Fernández-March, 2004: 128). 
La tarea de "aprender a aprender" conlleva la atenta reflexión de docentes y alumnos sobre los aprendizajes desarrollados. La hipótesis principal de este trabajo es que el portafolio o carpeta de aprendizaje del alumnado puede jugar un papel destacado en dicha reflexión y, además, constituye un instrumento adecuado para el aprendizaje autónomo del estudiante a través de lecturas y escritos reflexivos.

Por tanto, aquí se parte de una concepción constructivista del proceso de enseñanza-aprendizaje que requiere un enfoque concreto del hecho de enseñar. Tal enfoque consiste en crear las condiciones apropiadas para que los esquemas de conocimiento que construye el estudiante durante sus experiencias sean tan correctos, ricos y diversos como sea posible (Coll y Solé, 1989). La concepción constructivista del aprendizaje adquiere pleno sentido si se considera que éste no resulta total ni definitivamente fijado con la escucha atenta por los alumnos de la lección impartida por el profesor, puesto que la clase debe convertirse "en un espacio de aprendizaje, de formación activa [...] en un lugar en el que se participa activamente en la construcción del conocimiento" (Colen et al., 2006: 18). De hecho, se ha argumentado que no se "aprende por recepción pasiva: para apropiarse de un saber colectivo, los alumnos han de transformarlo" (Carlino, 2006: 153).

El portafolio, si se elabora adecuadamente por el alumno, puede ser una herramienta eficaz para que éste se apropie de los conocimientos. La efectiva realización del portafolio del alumno facilita dos tareas básicas: primero, que los discentes incorporen organizadamente los aprendizajes en relación con los objetivos planteados; $\mathrm{y}$, segundo, contribuye a que los docentes consigan información más detallada de los esquemas de conocimiento que usan los primeros y así poderles proporcionar la ayuda pedagógica adecuada (Colén et al., 2006: 30).

Al decir de Shulman el portafolio es una "historia documental estructurada de un conjunto (cuidadosamente seleccionado) de desempeños que han recibido preparación y tutoría, y [...] que sólo alcanzan realización plena en la escritura reflexiva, la deliberación y la conversación" (Shulman, 1999: 45). Si se aplica esta herramienta docente desde la perspectiva del aprendizaje del estudiante resulta en lo que se ha denominado "carpeta de aprendizaje del alumnado" (Colén et al., 2006) y/o "portafolios del alumnado" (García y Pozo, 2006; Rodríguez, 2011). El uso de dicha herramienta se sustenta sobre seis principios básicos: aporta una nueva perspectiva del aprendizaje, constituye un proceso en desarrollo, facilita el análisis de los logros y el aprendizaje, necesita autoevaluación, motiva la elección del estudiante y su reflexión sobre el trabajo e implica a los profesores como facilitadores del aprendizaje (Klenowski, 2005: 144).

En suma, el portafolio del alumnado permite establecer una relación intelectual entre discentes y docentes, de modo que ambos actores puedan participar en el proceso de construcción de los conocimientos. Igualmente, dicho portafolio ayuda al profesor a reflexionar sobre el aprendizaje global de cada alumno, de manera que el primero pueda ser más consciente de la evolución real de tal aprendizaje. Así, esta herramienta docente no es una simple colección de materiales, apuntes o esquemas, sino que puede constituirse en un mecanismo reflexivo para avanzar en la construcción activa del conocimiento. Por ello, el portafolio ha de integrar comentarios y/o reflexiones recíprocas de alumnos y profesores sobre las producciones de ambos (Rodríguez, 2011: 99), como así se muestra en este trabajo. 


\section{Metodología}

Para la debida contextualización de la experiencia aquí descrita se han de considerar los siguientes antecedentes. Primero, que entre los años 2007-2012, el autor de este trabajo hizo varios cursos de formación docente en el Espacio Europeo de Educación Superior (EEES), dentro del Plan Formativo del Profesorado de la Universidad Pablo de Olavide (Monreal y Ruiz, 2009: web). Segundo, que desde el curso 2009-2010, en el que se iniciaron las innovaciones docentes más adelante detalladas, se participó en las actividades de coordinación del Grado de Sociología de la Universidad Pablo de Olavide (UPO). Tercero, se desarrolló un estudio de viabilidad para la posible incorporación del portafolio al proceso de aprendizaje y evaluación de las Enseñanzas Prácticas y de Desarrollo (EPD) de la asignatura Introducción a la Ciencia Política (ICCP), perteneciente al Grado de Sociología de la UPO. Una vez elaborado el estudio de viabilidad, pareció conveniente incorporar el portafolio al proceso de aprendizaje y evaluación de la referida asignatura, desde octubre de 2010.

La asignatura ICCP tiene seis créditos europeos, lo cual equivale a una carga de trabajo para el estudiante de 150 horas, de las que 44 son horas presenciales en clase y las restantes se dedican a trabajo autónomo. Además, ésta es una asignatura obligatoria del primer curso del Grado de Sociología de la UPO, ubicada en el módulo de Formación Básica en Ciencias Sociales y en la que se matricularon 54 alumnos durante el curso 2011-2012.

En el marco de las Enseñanzas Prácticas y de Desarrollo (EPD) de la citada asignatura los estudiantes han de leer y comentar, a lo largo de nueve semanas, el libro $L a$ política como profesión de Max Weber (1864-1920). Al mismo tiempo, los alumnos han de elaborar un portafolio en el que reflexionen sobre las ideas que le sugiere dicho libro y así poder aprehender las prácticas discursivas de la Ciencia Política. Igualmente, se pretende que el alumno aproveche los conocimientos adquiridos en sus lecturas para pensar y reflexionar sobre los siguientes fenómenos políticos de los estados contemporáneos: la profesionalización de la política, la caracterización del estado como organización política y administrativa, el creciente poder de los partidos políticos, el liderazgo político democrático, el papel de los periodistas en política, los dilemas éticos del político profesional, etc.

Con la intención de promover la actitud reflexiva, la conversación, el trabajo colaborativo y autónomo se propone que se constituyan grupos de tres alumnos para seleccionar y comentar semanalmente noticias políticas publicadas en la prensa nacional o internacional. A los alumnos, se les proponen criterios concretos para seleccionar las noticias, que, en todo caso, han de estar referidas a los temas tratados en el citado libro de Max Weber.

Para este trabajo se seleccionan los discursos desarrollados en los portafolios de diez alumnos que constituyen una muestra intencional de los 54 estudiantes matriculados en la asignatura ICCP durante el curso 2011-2012. Los criterios para construir esta muestra intencional han sido: obtener una calificación superior a 2 puntos en las $\mathrm{EPD}$, integrar algunos alumnos que trabajen y estudien, tener otros estudios cursados y diversidad en las edades y sexos. El Cuadro 1, además de revelar estas características de los alumnos, contribuye a contextualizar los discursos que se analizan a continuación. 
Cuadro 1: Características de los alumnos y evaluación de sus aprendizajes (elaboración propia)

\begin{tabular}{|c|c|c|c|c|c|c|}
\hline $\begin{array}{c}\text { Alumno, } \\
\text { nombre en } \\
\text { clave }\end{array}$ & Edad & Sexo & $\begin{array}{c}\text { Otros estu- } \\
\text { dios cursa- } \\
\text { dos }\end{array}$ & $\begin{array}{c}\text { Trabaja } \\
\text { y estudia }\end{array}$ & $\begin{array}{c}\text { Sólo } \\
\text { estudia }\end{array}$ & $\begin{array}{c}\text { Evaluación de los } \\
\text { aprendizajes en } \\
\text { EPD* (de 0 a 4) }\end{array}$ \\
\hline JA & 20 & Varón & No & & $X$ & 4 \\
\hline JB & 46 & Varón & Sí & $X$ & & 2,4 \\
\hline JC & 18 & Mujer & No & & $X$ & 4 \\
\hline JD & 47 & Mujer & Sí & $X$ & & 3,1 \\
\hline JE & 22 & Varón & No & & $X$ & 4 \\
\hline JF & 21 & Varón & No & $X$ & & 3,3 \\
\hline JG & 18 & Mujer & No & & $X$ & 3,3 \\
\hline JH & 19 & Varón & No & & $X$ & 3,6 \\
\hline JI & 18 & Mujer & No & & $X$ & 3,3 \\
\hline JJ & 20 & Varón & No & $X$ & & 3,7 \\
\hline
\end{tabular}

*Aprendizajes evaluados con el portafolio del alumno

Por tanto, los discursos de los alumnos se extraen literalmente de los textos elaborados por ellos en sus portafolios. En estos textos los alumnos muestran su labor de lectura y escritura reflexiva durante su proceso de aprendizaje, lo cual es relevante para los objetivos del Espacio Europeo de Educación Superior (EEES), pues en este marco educativo se requiere un mayor protagonismo de los alumnos, así como que éstos sean motivados en el desarrollo de sus aprendizajes. En este sentido, se elabora un análisis de los discursos de los alumnos con la finalidad de conocer sus modos de construir los conocimientos. Entendiendo que construir los conocimientos implica, a su vez, los modos en que los alumnos organizan, elaboran, transforman, interpretan y codifican tales conocimientos. Como se mostrará seguidamente, los argumentos y evidencias construidas por los alumnos en sus portafolios confirman su proceso de aprendizaje autónomo mediante sus escritos reflexivos. Ello, sin duda, proporciona una información muy valiosa para el profesor y los estudiantes.

\section{Desarrollo}

La escritura y lectura reflexiva son mecanismos de aprendizaje autónomo no suficientemente bien aprovechados en el ámbito universitario (Carlino, 2006). De hecho, es una queja común entre los propios docentes universitarios confirmar el bajo nivel de escritura y de lectura de sus alumnos, incluso en los cursos más avanzados. Lo cual conlleva la necesidad de que los alumnos universitarios lean libros completos de sus respectivas disciplinas, de modo que puedan aprehender las prácticas discursivas desarrolladas en ellas y, a su vez, puedan construir sus conocimientos en relación con tales prácticas. Una razón para ello es que cuando los alumnos están iniciándose en una nueva disciplina, como sucede en esta experiencia, necesitan la guía del docente para conocer las claves discursivas de tal disciplina, pues:

"Leer y escribir son procesos intelectuales que se dan dentro de ciertas prácticas sociales: herramientas para aprender dependientes de modos culturales de hacer cosas con el 
lenguaje. [...] La autonomía [...] se adquiere para cierto ámbito cuando alguien está familiarizado con las prácticas que allí se llevan a cabo" (Carlino, 2006: 162-166).

Seguidamente se analizan un conjunto de escritos elaborados por los estudiantes de ICCP en sus portafolios. En éstos se observan: procesos de aprendizaje autónomo, evidencias sobre los trabajos grupales, intentos por incorporar las prácticas discursivas de la Ciencia Política y/o Teoría Política y el esfuerzo de los alumnos en construir sus conocimientos. En todo caso se mantendrá la confidencialidad de los discursos desarrollados por los estudiantes.

El discurso del alumno JA muestra cómo él fue afrontando y leyendo el libro propuesto. Este alumno se implicó bastante en el proceso de aprendizaje, lo cual se reveló mediante su planteamiento de dudas en clase y en tutoría, así como por otras lecturas específicas hechas por él autónomamente. Se puede observar que incorpora el tono reflexivo demandado:

"Me fascina el estilo metafísico y filosófico con el que Weber impregna sus páginas. Hace concebir la política como una rama del conocimiento y no como aquello que dan en las noticias antes de los deportes. En estos meses, las interminables conversaciones sobre la materia con personas de mi entorno me han formado en materia de política. [...] Empecé a comprender algunos conceptos fundamentales el último año de bachillerato pero no era aún algo sólido. Este verano, empecé a interesarme genuinamente por ella, leer periódicos, ver entrevistas en internet y a pensar con más detenimiento sobre esta caldeada actualidad que vivimos" (Alumno JA).

Este mismo alumno en la segunda entrada del portafolio, desarrollando su carácter reflexivo, muestra que algunas de sus ideas han surgido dentro del grupo en el que colaboró:

"Nuestro grupo ha reflexionado y debatido acerca de los dos billones de dólares que Estados Unidos invierte en presupuesto militar y que no están auditados [...] Si Estados Unidos empleara esa fortuna en labores humanitarias podrían perder defensa exterior contra otras potencias mundiales como China. Además de invertir ese dinero en combatir el hambre [...] se podía destinar a combatir la grave crisis que asola no sólo España sino el mundo" (Alumno JA).

El alumno JB aclara al inicio de su trabajo como éste es fruto del esfuerzo colectivo hecho en clase y en su grupo: "Las reflexiones vertidas en este trabajo son el fruto de las lecturas y de las charlas en reuniones con mis compañeros de grupo, además de las EPD realizadas para el análisis del texto con los asistentes a las clases y la guía del profesor" (Alumno JB).

Por su parte, la alumna JC empieza mostrando el espíritu reflexivo y selectivo que ella le imprime a su portafolio o diario de lecturas: "Este diario de lecturas recoge las reflexiones sobre los aspectos que más me han llamado la atención sobre cada una de las lecturas que he abordado. Las entradas no siguen un esquema común, pues he decidido escribir cada una de la forma con la que me fuese sintiendo más cómoda y con más recursos" (Alumna JC).

La alumna JC, tras mostrar un alto nivel de reflexividad en sus escritos, termina el portafolio con las siguientes apreciaciones que revelan su proceso de aprendizaje autónomo: 
"Cuando empecé a leer La política como profesión pensé que iba a ser un libro pesado de leer, y no me imaginaba terminando el diario de lecturas. Sin embargo, una vez inmersa en la lectura iba relacionando cosas, iba viendo detalles que me llevaban a la reflexión, a casos actuales, a noticias de hoy en día de las que ahora les encuentro una explicación, un porqué. En definitiva, Weber me ha acercado a la política, me ha hecho entenderla un poco [...] Me ha abierto los ojos al mundo de la política" (Alumna JC).

La alumna JD que actualmente trabaja, dispone de otra titulación universitaria y tiene 47 años, finaliza el portafolio con unas interesantes reflexiones sobre su aprendizaje autónomo que, a su vez, le permiten desmontar algunos prejuicios sobre política:

"leyendo este libro (muchas veces) he tenido que 'desaprender', he tenido que borrar de mi mente todos los prejuicios que tenía de políticos y sus políticas, con sentimientos de rechazo y desconfianza hacia todo el que se movía en este ambiente. He cambiado parte de mis prejuicios al ver que política es absolutamente todo lo que nos rodea, somos todos y todos hacemos política [...] La política está presente en todos nosotros desde antes de nacer" (Alumna JD).

El alumno JE muestra su pretensión de incorporar las prácticas discursivas de la Ciencia Política, que en parte consigue, si bien podría ser más preciso:

"Los medios de comunicación mediatizan la política. Estos medios de comunicación son una de las instituciones fundamentales dentro de cualquier sociedad moderna. Yo creo que los grandes periodistas son los grandes intelectuales dominantes en el mundo actual. Pero, también creo, que no son tantos los periodistas 'medios' los que dominan el mundo actual mediante la palabra sino los que dirigen a esos periodistas: los llamados 'magnates de la prensa'" (Alumno JE).

Tratando este mismo tema, de la relación entre política y prensa, el alumno JA hace unas reflexiones más precisas si cabe, que no desmerecen las elaboradas por el alumno JE.

"El análisis del periodismo en política nos lleva a observar la alargada figura del Magnate de la Prensa. Básicamente, éste cuenta con la libertad de publicar las noticias en función de sus intereses políticos y económicos. [...] Silvio Berlusconi, Jesús de Polanco o Pedro J. Ramírez tienen una influencia titánica en la política. Esto es algo muy negativo porque puede conllevar a una pérdida de la democracia [...] Además, el sociólogo alemán asegura que el político profesional está fielmente relacionado con el periodista y el magnate" (Alumno JA).

Asimismo, el alumno JF incorpora las prácticas discursivas de la Ciencia Política con bastante precisión, pues muestra ideas esclarecedoras que contribuyen a comprender la actividad política desde una perspectiva sociológica y normativa:

"La actividad organizada de la dominación (política) requiere una administración continuada y que para ello los hombres obedezcan órdenes y gracias a esta obediencia poder disponer de distintos recursos. Para este dominio se debe tener en cuenta al líder que administra las órdenes, pues se pueden cometer delitos humanos que vayan contra los derechos humanos (las purgas estalinistas, los campos de concentración nazis, etc.) [...] Por ello los poderes políticos tienen que estar divididos (como ocurre en una democracia)" (Alumno JF).

Por último, diversos alumnos coinciden en una valoración positiva de las lecturas y/o tareas que se les encomendó en la asignatura, aunque también comentan que al ini- 
cio les supuso gran esfuerzo. Pero las explicaciones de clase, las reflexiones grupales con sus compañeros, los ejemplos analizados y la labor de guía del profesor, han contribuido a que los alumnos empiecen a conocer el complejo mundo de la política.

"La política como profesión, me ha hecho reflexionar mucho sobre la política actual [...] He descubierto que Max Weber era un gran pensador [...] Me ha hecho adentrarme de una forma más profunda en el mundo de la política, y eso es algo de agradecer [...] También mencionar que el trabajo ha sido duro [...] y he tenido que estar tanto leyendo a Weber, como reflexionando sobre sus ideas, como informándome de esos conceptos que desconocía" (Alumna JG).

"El libro en general me ha parecido estupendo desde el punto de vista político, lógicamente, ya que me ha dado a entender numerosos hechos, conceptos, personajes, tipos de dominación, de políticos, de funcionarios especializados o periodistas, ... viendo más claro todo el mundo que me rodea [...]. Al principio, el libro es un poco pesado y denso, ya que estás tratando sobre un tema desconocido" (Alumno JH).

"Particularmente creo que cualquier político que aspire a hacer política debería leer esta obra y reflexionar sobre ella para saber lo que realmente tienen entre manos. Es más, incluso cualquier persona que sin ser político, le guste la política, le sería muy beneficiosa la lectura de este libro" (Alumna JI).

"El libro objeto de estudio no es cualquier libro sobre política, es El libro sobre política; una guía práctica $[\ldots]$ para todo aquel que pretenda ser un buen político [...]" (Alumno JJ).

\section{Conclusiones}

Anteriormente se presenta una experiencia docente de más de cinco años de preparación y estudio que ha tenido como principal objetivo enfocar el proceso de aprendizaje de los estudiantes en sus lecturas y escritos reflexivos. Mediante tales lecturas y escritos, los estudiantes de Ciencia Política muestran diversas reflexiones sugeridas por textos clásicos de esta disciplina y, a su vez, ello les permite construir autónomamente los conocimientos. Ambas tareas son fundamentales para centrarse en los aprendizajes del alumnado, los cuales se revelan en sus portafolios. No obstante, para poder mejorar este proceso de aprendizaje, es preciso mostrar sus ventajas e inconvenientes.

Se aprecian cinco ventajas. Primero, los alumnos implicados en el proceso de trabajo descrito han estado muy motivados en su aprendizaje, como así revelan sus discursos. Segundo, como los alumnos se sienten motivados en lo que van aprendiendo y ello lo deben reflejar en su portafolio, éste, a su vez, se convierte en un registro eficaz del proceso de aprendizaje autónomo. Tercero, el hecho de que los alumnos/as hayan formado grupos de trabajo en las EPD, sirve para que pongan ideas en común sobre su trabajo individual y planteen diversas dudas. Cuarto, el portafolio es una herramienta muy útil para desarrollar un aprendizaje autónomo en el que el estudiante madure progresivamente los conceptos, teorías y textos objeto de estudio. Así, el alumno advierte que lo importante no es asimilar conocimientos pasivamente, sino integrarlos e incorporarlos en su proceso de trabajo personal. Quinto, en el trabajo grupal de clase se comprueba que se puede mantener un contacto más cercano con los estudiantes, lo cual facilita que éstos manifiesten sus dudas y/o reflexiones comunes.

Se observan cinco posibles inconvenientes. En primer lugar, el alumno que no puede asistir a alguna sesión presencial de Enseñanzas Prácticas y de Desarrollo (EPD) se puede ver descolgado del proceso de aprendizaje. En segundo lugar, se comprueba que aquellos alumnos que asisten irregularmente a las EPD es difícil hacerles un 
seguimiento de su trabajo autónomo. En tercer lugar, el referido sistema de enseñanzaaprendizaje es muy dependiente de la interacción presencial de los alumnos con el profesor, y viceversa. En cuarto lugar, para aquellos alumnos que no asisten a las clases no se plantea un sistema alternativo de trabajo. Por último, aparece la dificultad de evaluar el trabajo colaborativo de cada grupo de alumnos.

\section{Referencias bibliográficas}

BENITO, Águeda y CRUZ, Ana (2005): Nuevas claves para la Docencia Universitaria en el Espacio Europeo de Educación Superior. Madrid, Narcea.

CARLINO, Paula (2006): Escribir, leer y aprender en la Universidad. Una introducción a la alfabetización académica. Buenos Aires, Fondo de Cultura Económica.

COLÉN, María Teresa et al. (2006): La carpeta de aprendizaje del alumnado universitario. La autonomía del estudiante en el proceso de aprendizaje. Barcelona, Octaedro-ICE.

COLL, César y SOLÉ, Isabel (1989): “Aprendizaje significativo y ayuda pedagógica". Cuadernos de Pedagogía, no 168, pp. 16-20.

FERNÁNDEZ-MARCH, Amparo (2004): "El portafolio docente como estrategia formativa y de desarrollo profesional". Educar, n 33 , pp. 127-142.

GARCÍA, Beatriz y POZO, María Teresa (2006): "El portafolios del alumnado: una investigación-acción en el aula universitaria". Revista de Educación, n 341, pp. 737-756.

KLENOWSKI, Val (2005): Desarrollo del Portafolios para el aprendizaje y la evaluación. Procesos y principios. Madrid, Narcea.

MONREAL, María del Carmen y RUIZ, Esteban (2009): "La Formación del Profesorado en el marco de la innovación docente universitaria. El caso de la Universidad Pablo de Olavide", en Red-U, Revista de Docencia Universitaria, $\mathrm{n}^{\circ} 4$ : http://redaberta.usc.es/redu/index.php/REDU/article/view/114.

RODRÍGUEZ, Manuel (2011): "Metodologías docentes en el EEES: de la clase magistral al portafolio". Tendencias Pedagógicas, nº 17, pp. 83-103.

SHULMAN, Lee (1999): "Portafolios del docente: una actividad teórica", en LYONS, Nona: El uso del portafolios. Propuestas para un nuevo profesionalismo docente. Buenos Aires, Amorrortu, pp. 45-62.

\section{José Francisco JIMÉNEZ DÍAZ}

josefco@upo.es

Carretera de Utrera, Km. 1, CP: 41013, Sevilla

Universidad Pablo de Olavide.

Profesor Contratado Doctor

Área de Ciencia Política y de la Administración

Departamento de Derecho Público

Facultad de Derecho. 